\title{
ON COMPLETE CONGRUENCE LATTICES OF COMPLETE LATTICES
}

\author{
G. GRÄTZER AND H. LAKSER
}

\begin{abstract}
The lattice of all complete congruence relations of a complete lattice is itself a complete lattice. In this paper, we characterize this lattice as a complete lattice. In other words, for a complete lattice $L$, we construct a complete lattice $K$ such that $L$ is isomorphic to the lattice of complete congruence relations of $K$. Regarding $K$ as an infinitary algebra, this result strengthens the characterization theorem of congruence lattices of infinitary algebras of G. Grätzer and W. A. Lampe. In addition, we show how to construct $K$ so that it will also have a prescribed automorphism group.
\end{abstract}

\section{INTRODUCTION}

In 1983, R. Wille raised the following question [24]: Is every complete lattice isomorphic to the lattice of complete congruence relations of a suitable complete lattice? See also K. Reuter and R. Wille [20].

A closely related question was raised by G. Birkhoff [1] in 1945: Is every complete lattice isomorphic to the lattice of congruence relations of a suitable (infinitary) algebra? In 1948, Birkhoff restated this question in the second edition of his "Lattice theory" [2]; however, "(infinitary)" was dropped from the question. This was intentional; Birkhoff referred to some continuity conditions that must hold in a congruence lattice of a (finitary) algebra.

The finitary problem was solved by G. Grätzer and E. T. Schmidt [15] in 1961. The congruence lattice of a (finitary) algebra was characterized as an algebraic lattice. The proof was based on a transfinite sequence of constructions starting out with a partial algebra with the required congruence lattice and some special properties, and repeating two steps:

(1) Form the free extension of the partial algebra.

(2) Introduce partial operations on the free algebra to "kill" all congruences that do not arise naturally as congruences extended from the partial algebra.

Received by the editors September 19, 1988 and, in revised form February 6, 1990.

1980 Mathematics Subject Classification (1985 Revision). Primary 08A65, 06A23; Secondary 08A30, 06B10.

Key words and phrases. Complete lattice, congruence lattice, complete congruence, automorphism group.

This research was supported by the NSERC of Canada. 
The difficulty lay in showing that the special properties one started with were preserved. A number of alternative proofs appeared. However, none provided a direct construction; see, for instance, E. T. Schmidt [22], in 1973, W. A. Lampe [16], and in 1976, P. Pudlák [18].

Grätzer and Lampe [13] announced a solution to the infinitary case in 19711972, and obtained, in particular, an affirmative answer to Birkhoff's 1945 question. Since the algebra to be constructed was infinitary, the two step transfinite construction became even more complex; see Appendix 7 of [7] for full details. See also E. Nelson [17].

Considering the complexity of this proof, it was with some surprise that the first author discovered ${ }^{1}$ that the answer to the question of Wille is in the affirmative:

Theorem 1. Every complete lattice $L$ is isomorphic to the lattice of complete congruence relations of a suitable complete lattice $K$.

We present in this paper a proof of this theorem, which is much simpler and cleaner than the original proof of Grätzer (unpublished), and yields a lattice of much simpler structure.

This result is a much stronger form of the infinitary case of the result of Grätzer and Lampe: $K$ is a complete lattice, while Grätzer and Lampe constructed an infinitary algebra of unspecified type. The construction is simple enough to be presented in a few diagrams along with some explanations. The lattice $K$ is "planar" (i.e., of order-dimension 2). The proof uses a one step construction, doing away with partial algebras, free algebras, and the transfinite sequence of constructions. On the other hand, Grätzer and Lampe handle the finitary and infinitary case together. The present method is infinitary in nature; it does not apply to the finitary case.

A partial solution ( $L$ was assumed to be a complete distributive lattice in which every element was the (infinite) join of (finitely) join-irreducible elements) was presented by Reuter and Wille in [20]. The construction of Reuter and Wille yielded a finite lattice $K$ for a finite lattice $L$. Hence their result was a generalization of a theorem of R. P. Dilworth: Every finite distributive lattice $L$ is isomorphic to the congruence lattice of some finite lattice $K$.

The proof of Dilworth's result was first published in Grätzer and Schmidt [14]. A somewhat different proof, due to Grätzer and H. Lakser, appeared in [6, pp. 81-84].

The proof of Reuter and Wille was based on an earlier paper of Wille [24] on complete congruence relations of concept lattices. In [12], Grätzer, Lakser, and B. Wolk showed how the approaches of [6] and [14] also apply.

\footnotetext{
${ }^{1}$ The results of this paper were announced by the first author on June 25,1988 , at an invited lecture at the International Conference on Universal Algebra, Lattices, and Semigroups, at the Centro de Algebra, Instituto Nacional de Investigação Científica, Universidade de Lisboa. See also the announcement in G. Grätzer [8].
} 
A very important partial solution to the question of Wille was obtained in S.-K. Teo [23]: Every finite lattice $L$ is isomorphic to the lattice of complete congruence relations of a suitable complete lattice $K$. The coloring construction for chains used in this paper originated in Teo [23].

For infinitary algebras, Grätzer and Lampe [13] (see also Appendix 7 of [7]) proved a much stronger result combining congruences with automorphisms. The first author announced in Grätzer [8] that their result could be strengthened the same way as Theorem 1 strengthened their characterization of congruence lattices of infinitary algebras.

Theorem 2. Let $L$ be a complete lattice with more than one element. Let $G$ be an arbitrary group. Then there exists a complete lattice $K$ such that the lattice of complete congruence relations of $K$ is isomorphic to $L$, and the automorphism group of $K$ is isomorphic to $G$.

Our proof of Theorem 2 is much simpler than the (unpublished) proof of Grätzer.

The basic notation is explained in $\S 2$. In $\S 3$, we introduce the coloring $\varphi$ of a chain $C$, and investigate the complete congruences of the associated expansion $C(\varphi)$. The crucial construction, which we call the $\mathfrak{M}_{X}$ construction, is discussed in $\S 4$; this, basically, guarantees that for a subset $X$ of $L-\{0\}$, the complete congruences associated with $x \in X$ join to the complete congruence associated with $\bigvee X$. In $\S 5$, we construct a chain $X^{\dagger}$ from a nonempty subset $X$ of $L-\{0\}$. From all such chains $X^{\dagger}$, we build the chain $C$, and define a natural coloring $\varphi$ of $C$. We add some elements to the expansion $C(\varphi)$ to obtain, in $C(\varphi)$, a copy of $\mathfrak{M}_{X}$, for each $X$; the resulting lattice will be the lattice $K$ of Theorem 1. In $\S 6$, we investigate the complete congruences of $K$, and prove Theorem 1 . In $\S 7$, we show how to extend $K$ to accomodate an arbitrary automorphism group to provide a proof of Theorem 2 . $\S 8$ contains some comments on the construction.

\section{NotATION}

$L$ is the complete lattice we want to represent in Theorem 1 . 0 and 1 denote the zero and unit element of $L$, respectively. If $|L|=1$, it is trivial to represent $L$. We shall, henceforth, assume that $|L| \geq 2$.

We shall be dealing with nonempty subsets $X \subseteq L-\{0\}$. Let us write

$$
X=\left\{x_{\gamma} \mid \gamma<\zeta^{X}\right\}
$$

where $1 \leq \zeta^{X} \leq \zeta=|L-\{0\}|$. Let

$$
\left\{X^{\delta} \mid \delta<\chi\right\}
$$

denote the family of all such sets; the elements of $X^{\delta}$ are well-ordered:

$$
X^{\delta}=\left\{x_{\gamma}^{\delta} \mid \gamma<\zeta^{\delta}\right\}
$$

Since $|L| \geq 2$, it follows that there is at least one such $X$, that is, $0<\chi$. 
$\mathfrak{M}_{3}$ denotes the five-element modular nondistributive lattice. $\mathfrak{C}_{n}$ is the $n$ element chain with elements $0,1, \ldots, n-1$.

Let $\alpha$ be an ordinal, and for $\gamma<\alpha$, let $A_{\gamma}$ be a lattice. We denote by $\sum\left(A_{\gamma} \mid \gamma<\alpha\right)$ the ordinal sum of the $A_{\gamma}$ for $\gamma<\alpha$; for two components, $A$ and $B, A+B$ denotes the ordinal sum of $A$ and $B$ (we place $B$ on top of $A)$.

For ordinals $\alpha, \beta$, the ordinal product $\alpha \times \beta$ is regarded as the set $\{\langle\gamma, \delta\rangle \mid$ $\gamma<\alpha, \delta<\beta\}$ ordered by $\left\langle\gamma_{1}, \delta_{1}\right\rangle \leq\left\langle\gamma_{2}, \delta_{2}\right\rangle$ iff $\gamma_{1}<\gamma_{2}$ or $\gamma_{1}=\gamma_{2}$ and $\delta_{1} \leq \delta_{2}$.

For a lattice $A$, let Ip $A$ denote the the set of prime intervals in $A$, i.e., the set of all intervals $\mathfrak{p}=[u, v]$, where $u \prec v$. If $\mathfrak{p}=[u, v] \in \operatorname{Ip} A$, then for any lattice $B$ and $b \in B$, we use the notation

$$
\mathfrak{p} \times\{b\}=[\langle u, b\rangle,\langle v, b\rangle] \in \operatorname{Ip}(A \times B) .
$$

If $\mathfrak{p}_{1}=\left[x_{1}, y_{1}\right] \in \operatorname{Ip} A_{1}$ and $\mathfrak{p}_{2}=\left[x_{2}, y_{2}\right] \in \operatorname{Ip} A_{2}$, then it will be convenient to refer to the elements of the four-element sublattice of $A \times B$ generated by $\mathfrak{p}_{1} \times\left\{x_{2}\right\}$ and $\left\{x_{1}\right\} \times \mathfrak{p}_{2}$ as follows:

$$
\begin{aligned}
& o\left(\mathfrak{p}_{1}, \mathfrak{p}_{2}\right)=\left\langle x_{1}, x_{2}\right\rangle, \quad a\left(\mathfrak{p}_{1}, \mathfrak{p}_{2}\right)=\left\langle y_{1}, x_{2}\right\rangle, \\
& b\left(\mathfrak{p}_{1}, \mathfrak{p}_{2}\right)=\left\langle x_{1}, y_{2}\right\rangle, \quad i\left(\mathfrak{p}_{1}, \mathfrak{p}_{2}\right)=\left\langle y_{1}, y_{2}\right\rangle .
\end{aligned}
$$

If $A_{1}=A_{2}=A$, and $\mathfrak{p}_{1}=\left[x_{1}, y_{1}\right], \mathfrak{p}_{2}=\left[x_{2}, y_{2}\right] \in \operatorname{Ip} A$, the notation $o\left(\mathfrak{p}_{1}, \mathfrak{p}_{2}\right), a\left(\mathfrak{p}_{1}, \mathfrak{p}_{2}\right), \quad b\left(\mathfrak{p}_{1}, \mathfrak{p}_{2}\right), i\left(\mathfrak{p}_{1}, \mathfrak{p}_{2}\right)$ refer to the four elements in $A^{2}$, where $\mathfrak{p}_{1}$ is regarded as a prime interval of the first component, and $\mathfrak{p}_{2}$ is regarded as a prime interval of the second component.

For a (prime) interval $\mathfrak{p}=[u, v]$ in the lattice $A$, we shall denote by $\Theta_{A}(\mathfrak{p})$ or $\Theta_{A}(u, v)$ the congruence relation generated by the prime interval $\mathfrak{p}$. If $A$ is understood, we use the notation $\Theta(\mathfrak{p})$ or $\Theta(u, v)$. In case $A$ is complete, $\Theta_{A}^{c}(\mathfrak{p})$ or $\Theta_{A}^{c}(u, v)$ (or, simply, $\Theta^{c}(\mathfrak{p})$ or $\Theta^{c}(u, v)$ ) will denote the complete congruence relation generated by $\mathfrak{p}=[u, v]$. Note that $u \equiv v(\Theta)$ is equivalent to $\Theta(\mathfrak{p}) \leq \Theta$.

For a lattice $A$, the congruence lattice of $A$ is denoted by Con $A$; the lattice operations in Con $A$ are denoted by $\wedge, \vee$, and the infinite variants by $\Lambda$, $\checkmark$. For a complete lattice $A$, the complete congruence lattice of $A$ is denoted by $\operatorname{Con}^{c} A$; the lattice operations in $\operatorname{Con}^{c} A$ are denoted by $\wedge, \vee^{c}$, and the infinite variants by $\Lambda, V^{c}$. Notice that there is no change in notation for meets (meets are set intersections); for complete congruences $\Theta_{i}, i \in I$, the complete join, $\bigvee^{c}\left(\Theta_{i} \mid i \in I\right)$ is the smallest complete congruence of $A$ containing $\mathrm{V}\left(\boldsymbol{\Theta}_{i} \mid i \in I\right)$.

We refer the reader to [6] for the standard notation in lattice theory.

\section{COLORING}

A coloring of a chain $C$ is a map

$$
\varphi: \operatorname{Ip} C \rightarrow L-\{0\} .
$$




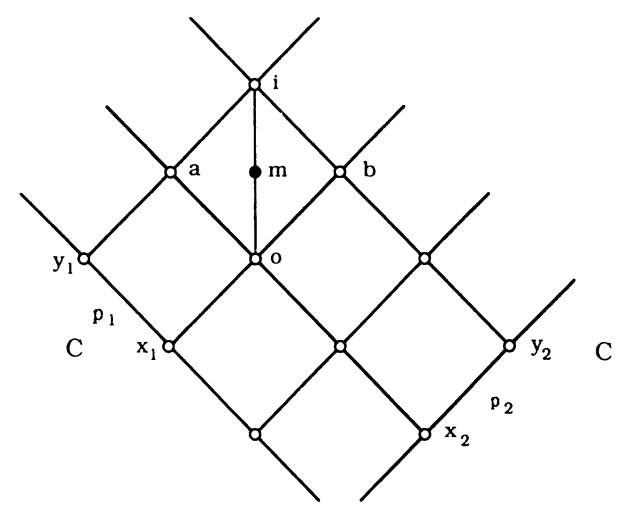

FIGURE 1

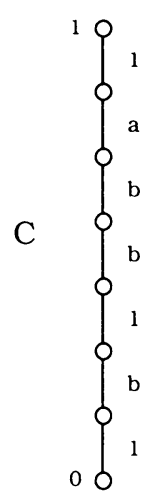

FIGURE 2

If $\mathfrak{p} \in \operatorname{Ip} C$ and $\mathfrak{p} \varphi=a$, one should think of $\Theta_{K}^{c}(\mathfrak{p})$ as the complete congruence representing $a \in L-\{0\}$ in some extension $K$ of $C$.

Following Teo [23], for a chain $C$ and coloring $\varphi$, we define the lattice $C(\varphi)$ as follows: the lattice $C(\varphi)$ is $C^{2}$ augmented with the elements $m\left(\mathfrak{p}_{1}, \mathfrak{p}_{2}\right)$, whenever $\mathfrak{p}_{1}, \mathfrak{p}_{2} \in \operatorname{Ip} C$ and $\mathfrak{p}_{1} \varphi=\mathfrak{p}_{2} \varphi$, and we require that the elements

$$
o\left(\mathfrak{p}_{1}, \mathfrak{p}_{2}\right), a\left(\mathfrak{p}_{1}, \mathfrak{p}_{2}\right), \quad b\left(\mathfrak{p}_{1}, \mathfrak{p}_{2}\right), m\left(\mathfrak{p}_{1}, \mathfrak{p}_{2}\right), i\left(\mathfrak{p}_{1}, \mathfrak{p}_{2}\right)
$$

form a sublattice of $C(\varphi)$ isomorphic to $\mathfrak{M}_{3}$, as illustrated by Figure 1, where $\mathfrak{p}_{1}=\left[x_{1}, y_{1}\right]$ and $\mathfrak{p}_{2}=\left[x_{2}, y_{2}\right]$. Obviously, $C(\varphi)$ is an extension of $C$. If $C$ is complete, so is $C(\varphi)$.

For instance, let $C$ be the chain of Figure 2, where the name of the elements appear to the left of the $\circ$ and the color of a prime interval appears to the right of the edge. Figure 3 illustrates $C(\varphi)$.

The congruences of $C^{2}$ are of the form $\Theta_{1} \times \Theta_{2}$, where $\Theta_{1}$ and $\Theta_{2}$ are congruences of $C$. Now take only $\Theta_{1}$ and $\Theta_{2}$ with the following property: (3-2) If $\mathfrak{p}_{1}, \mathfrak{p}_{2} \in \operatorname{Ip} C$ and $\mathfrak{p}_{1} \varphi=\mathfrak{p}_{2} \varphi$, then

$$
\Theta\left(\mathfrak{p}_{1}\right) \leq \Theta_{1} \quad \text { iff } \quad \Theta\left(\mathfrak{p}_{2}\right) \leq \Theta_{2} \text {. }
$$


Now we extend the congruence $\Theta_{1} \times \Theta_{2}$ on $C^{2}$ to $C(\varphi)\left(\supseteq C^{2}\right)$ as follows: for $\mathfrak{p}_{1}, \mathfrak{p}_{2} \in \operatorname{Ip} C$ and $\mathfrak{p}_{1} \varphi=\mathfrak{p}_{2} \varphi$, let the elements in (3-1) be in one congruence class. Let $\Theta_{1} \times{ }_{\varphi} \Theta_{2}$ denote this extension. It is easy to compute that the congruences of $C(\varphi)$ are exactly the congruences of the form $\Theta_{1} \times{ }_{\varphi} \Theta_{2}$.

In the special case $\Theta=\Theta_{1}=\Theta_{2}$, we shall use the notation $\Theta(\varphi)$ for the congruence $\Theta \times{ }_{\varphi} \Theta$.

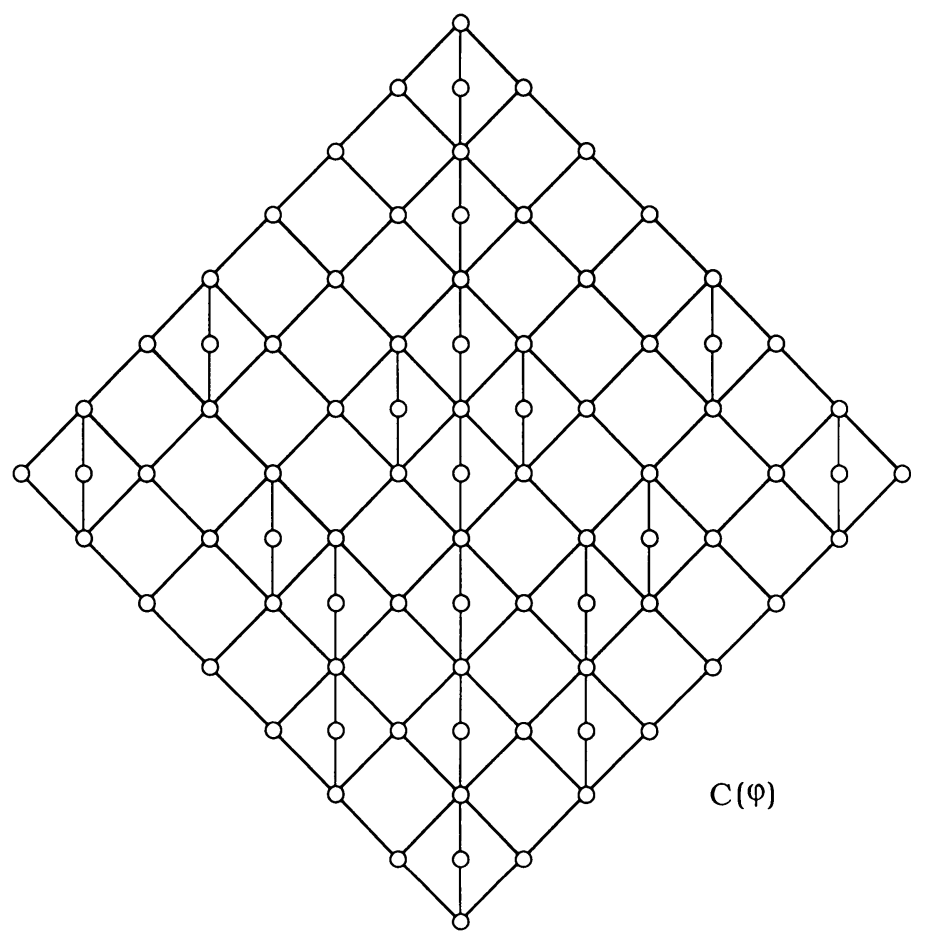

FIGURE 3

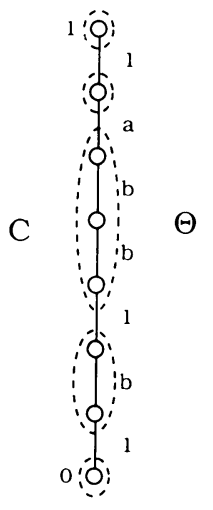

FIGURE 4 


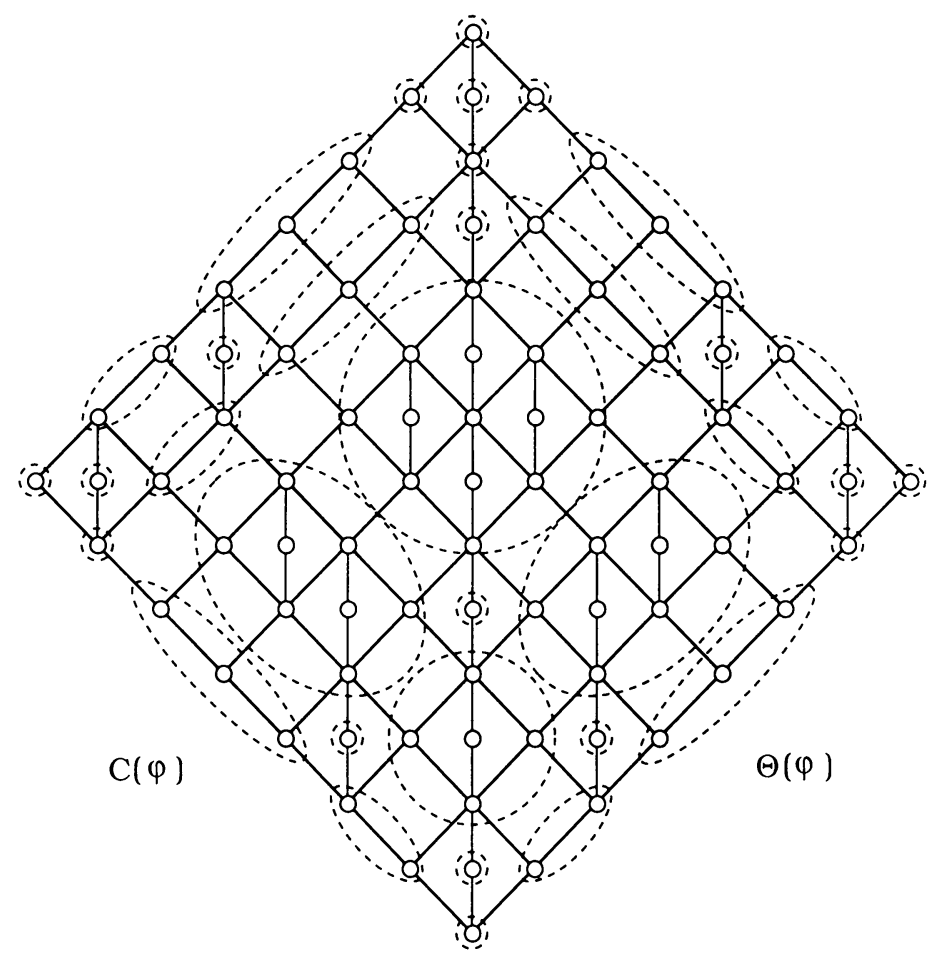

FIGURE 5

By taking $\mathfrak{p}_{1}=\mathfrak{p}_{2}$, we observe that $\Theta_{1}$ and $\Theta_{2}$ collapse exactly the same prime intervals of $C$. Thus, for a finite $C$, we have $\Theta_{1}=\Theta_{2}$; the congruences of $C(\varphi)$ are of the form $\Theta(\varphi)$, where $\Theta$ is a congruence of $C$ with property (3-2).

As an example, take the congruence $\Theta$ of the chain $C$ of Figure 2, as shown in Figure 4. Then $\Theta(\varphi)$ is the congruence of $C(\varphi)$ as illustrated by Figure 5.

To handle the infinite case, for a complete lattice $A$ and a complete congruence $\Theta$ on $A$, we define the prime interior of $\Theta$, pi $\Theta$, as follows:

$$
\operatorname{pi} \Theta=\bigvee^{c}\left(\Theta^{c}(\mathfrak{p}) \mid \mathfrak{p} \in \operatorname{Ip} A, \Theta(\mathfrak{p}) \leq \Theta\right) \text {. }
$$

For the complete congruences $\Theta_{1}$ and $\Theta_{2}$ on the complete chain $C$, the relation $\Theta_{1} \times{ }_{\varphi} \Theta_{2}$, defined above on $C(\varphi)$, is a complete congruence of $C(\varphi)$ iff condition (3-2) holds, in which case pi $\Theta_{1}=$ pi $\Theta_{2}$.

Let $A$ be a complete lattice which is strongly atomic, that is, for any $w, z \in$ $A, w<z$, there is an element $p \in A$ satisfying $w \prec p \leq z$. In a strongly atomic complete lattice, there are very many complete congruences generated by prime intervals:

Lemma 1. In a strongly atomic complete lattice $A$, the equality

$$
\text { pi } \Theta=\Theta
$$

holds for any complete congruence $\Theta$ of $A$. 
Proof. The inclusion pi $\Theta \leq \Theta$ is obvious. Conversely, let $x \leq y$ and $x \equiv y$ $(\Theta)$; we wish to prove that

$$
x \equiv y \quad(\mathrm{pi} \Theta) .
$$

Since pi $\Theta$ is a complete congruence, it follows that there exists a maximal element $z$ in $[x, y]$ satisfying $x \equiv z(\mathrm{pi} \Theta)$. If $z=y$, we are done. Otherwise, $z<y$, so, by the strong atomicity of $A$, there exists an element $p$ with $z \prec p \leq y$. By $x \equiv y(\Theta)$, it follows that $z \equiv p(\Theta)$. Since $[z, p]$ is prime, it follows from the definition of pi $\Theta$ that $z \equiv p(\mathrm{pi} \Theta)$. Thus, $x \equiv p(\mathrm{pi} \Theta)$, contradicting the maximality of $z$.

Combining Lemma 1 with the discussion of the congruences of $C(\varphi)$, we obtain:

Lemma 2. Let $C$ be a complete strongly atomic chain. Then the complete congruences of $C(\varphi)$ are the congruences of the form $\Theta(\varphi)$, where $\Theta$ is a complete congruence of $C$ satisfying (3-2) with $\Theta=\Theta_{1}=\Theta_{2}$, that is, if $\mathfrak{p}_{1}, \mathfrak{p}_{2} \in \operatorname{Ip} C$, $\mathfrak{p}_{1} \varphi=\mathfrak{p}_{2} \varphi$, and $\mathfrak{p}_{1}$ is collapsed by $\Theta$, then $\mathfrak{p}_{2}$ is collapsed by $\Theta$.

We shall leave the routine, though somewhat tedious, verification of this lemma to the reader.

\section{The LATtice $\mathfrak{M}_{X}$}

Let $X=\left\{x_{\gamma} \mid \gamma<\zeta^{X}\right\} \subseteq L-\{0\}$ be given as in (2-1).

First, we construct a chain $X^{\dagger}$, then the lattice $\mathfrak{M}_{X}$. The chain $X^{\dagger}$ is defined (see Figure 6) as the chain

$$
\mathfrak{C}_{1}+(\omega \times X)+\mathfrak{C}_{1} .
$$

The elements of $X^{\dagger}$ are denoted as follows: the zero and unit elements are $0^{X}$ and $1^{X}$, respectively (for $X^{\delta}$, we use $0^{\delta}$ and $1^{\delta}$ ); the other elements are

$$
j^{X}=\left\langle 0, x_{0}\right\rangle<\left\langle 0, x_{1}\right\rangle<\cdots<\left\langle i, x_{0}\right\rangle<\left\langle i, x_{1}\right\rangle<\cdots,
$$

for $i<\omega$.

We define a coloring $\varphi^{X}$ (for $X^{\delta}$, denoted by $\varphi^{\delta}$ ) on $X^{\dagger}$ as illustrated by Figure 6:

$$
\begin{aligned}
{\left[0^{X}, j^{X}\right] \varphi^{X} } & =\bigvee X ; \\
{\left[\left\langle i, x_{\gamma}\right\rangle, u\right] \varphi^{X} } & =x_{\gamma} \quad \text { for } i<\omega \text { and } \gamma<\zeta^{X},
\end{aligned}
$$

where

$$
u= \begin{cases}\left\langle i, x_{\gamma+1}\right\rangle, & \text { if } \gamma+1<\zeta^{X} \\ \left\langle i+1, x_{0}\right\rangle, & \text { if } \gamma+1=\zeta^{X} .\end{cases}
$$

Note that all the prime intervals are accounted for, so this is a complete definition of a coloring.

The following observation is trivial but crucial: 


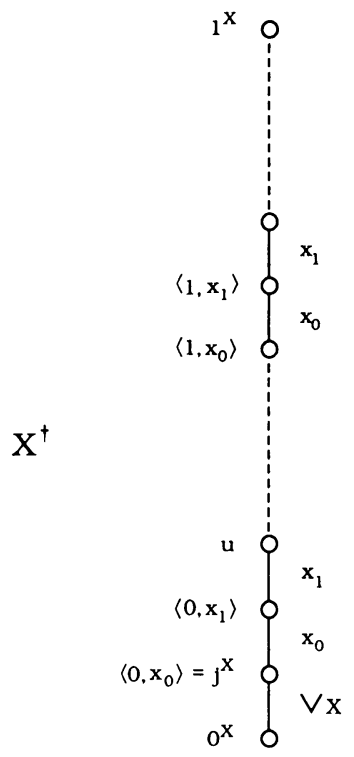

\section{FIGURE 6}

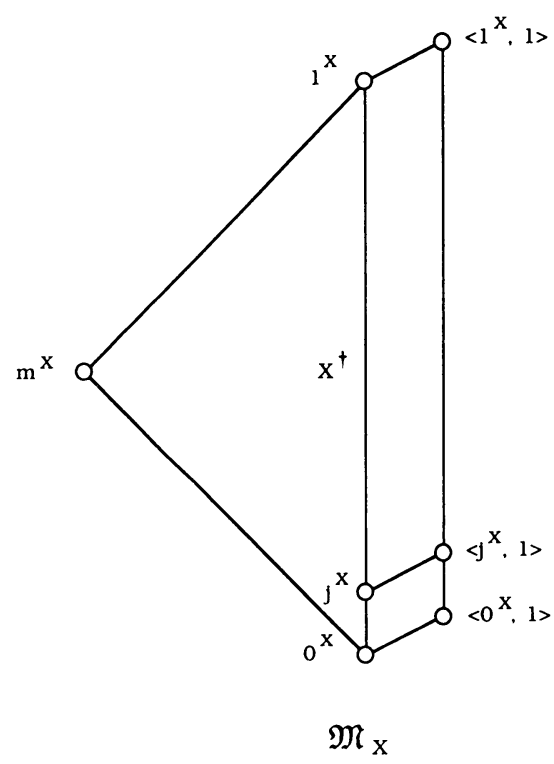

FIGURE 7

Lemma 3. The chain $X^{\dagger}$ is well-ordered, and $1^{X}$ is a limit. In $X^{\dagger}$, for every $j^{X} \leq u<1^{X}$ and for every $\gamma<\zeta^{X}$, there is a prime interval $\mathfrak{p}$ in $\left[u, 1^{X}\right]$ such that $\mathfrak{p} \varphi=x_{\gamma}$.

Now we define the lattice $\mathfrak{M}_{X}$ (and $\mathfrak{M}_{X^{\delta}}$ ) (see Figure 7). First, we form $X^{\dagger} \times \mathfrak{C}_{2}$. For $x \in X^{\dagger}$, we identify $\langle x, 0\rangle$ with $x$. (We make the same 
identification in $\left(X^{\delta}\right)^{\dagger} \times \mathfrak{C}_{2}$, for $\delta<\chi$.) This makes $X^{\dagger}$ a complete sublattice of $X^{\dagger} \times \mathfrak{C}_{2}$. Then we form $\mathfrak{M}_{X}$ by adding to $X^{\dagger} \times \mathfrak{C}_{2}$ the element $m^{X}$ (denoted by $m^{\delta}$ for $X^{\delta}$ ) satisfying

$$
0^{X} \prec m^{X} \prec 1^{X} .
$$

Obviously, $\mathfrak{M}_{X}$ is a complete lattice, and $X^{\dagger}$ is a complete sublattice of $\mathfrak{M}_{X}$.

\section{The LatTice $K$}

Now we are ready to construct the lattice $K$ of Theorem 1. For every $X^{\delta}$, $\delta<\chi$, we construct the chain $\left(X^{\delta}\right)^{\dagger}$ and form the ordinal sum:

$$
C= \begin{cases}\mathfrak{C}_{1}+\sum\left\{\left(X^{\delta}\right)^{\dagger} \mid \delta<\chi\right\}+\mathfrak{C}_{1}, & \text { if } \chi \text { is limit; } \\ \mathfrak{C}_{1}+\sum\left\{\left(X^{\delta}\right)^{\dagger} \mid \delta<\chi\right\}, & \text { if } \chi \text { is not limit }\end{cases}
$$

(see Figure 8). The zero and unit elements of $C$ are denoted by $0^{C}$ and $1^{C}$, respectively. Observe that if $\chi$ is not limit, that is, $\chi=\chi_{0}+1$, then $1^{C}=1^{\chi_{0}}$.

$C$ is a well-ordered chain. In (5-1), we distinguished the two cases to make sure that the unit element is a limit; this plays a role in the proof that $K$ has no nontrivial automorphism (see Lemma 14).

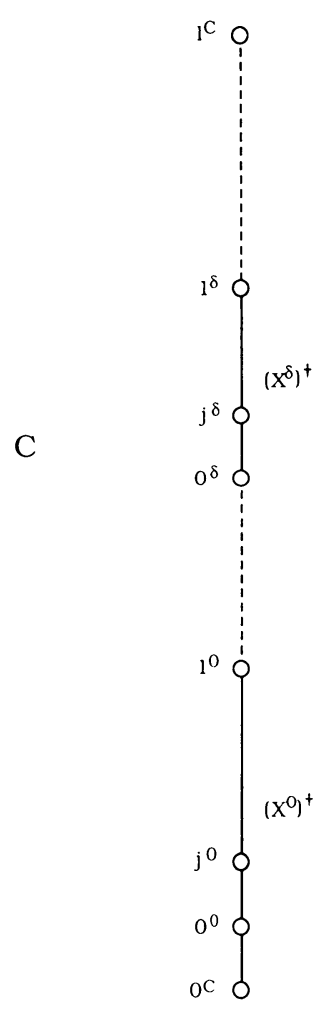

FIGURE 8 


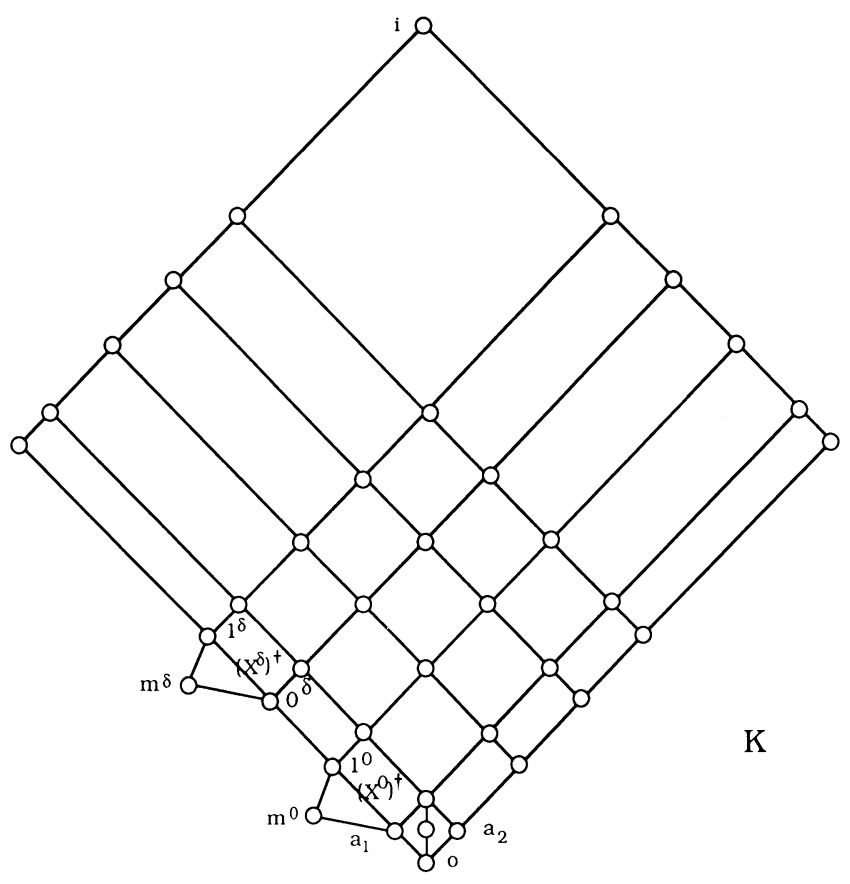

FigURE 9

Next, we define a coloring $\varphi$ of $C$. For a prime interval $\mathfrak{p}$ of $C$, let

$$
\mathfrak{p} \varphi= \begin{cases}\mathfrak{p} \varphi^{\delta}, & \text { if } \mathfrak{p} \in \operatorname{Ip}\left(X^{\delta}\right), \text { for some } \delta<\chi ; \\ 1, & \text { if } \mathfrak{p}=\left[0^{C}, 0^{0}\right] ; \\ 1, & \text { if } \mathfrak{p}=\left[1^{\delta}, 0^{\delta+1}\right], \text { for some } \delta<\chi .\end{cases}
$$

Reading the proofs in $\S \S 5$ and 6 , the reader will see that, for $\delta<\chi$, the color $\left[1^{\delta}, 0^{\delta+1}\right] \varphi$ could have been defined as any element of $L-\{0\}$. Also, as an alternative, in the definition of $C$, for $\delta<\chi$, we could have identified $1^{\delta}$ with $0^{\delta+1}$, thereby eliminating all prime intervals of the form $\left[1^{\delta}, 0^{\delta+1}\right]$.

Finally, we define the lattice $K$ as $C(\varphi)$ augmented with the elements $m^{\delta}$, for $\delta<\chi$ (see Figure 9).

More formally,

$$
K=C(\varphi) \cup\left\{m^{\delta} \mid \delta<\chi\right\}
$$

partially ordered as follows, where $x, y$ denote elements of $C(\varphi)$ :

$$
\begin{aligned}
& x \leq y \text { retains its meaning in } C(\varphi) \text {; } \\
& m^{\delta}<x \quad \text { iff }\left\langle 1^{\delta}, 0^{C}\right\rangle \leq x \text { in } C(\varphi) \text {, for } \delta<\chi \text {; } \\
& x<m^{\delta} \text { iff } x \leq\left\langle 0^{\delta}, 0^{C}\right\rangle \text { in } C(\varphi) \text {, for } \delta<\chi \text {. }
\end{aligned}
$$

It is easy to see that $K$ is a complete lattice, $C(\varphi)$ is a complete $\{0,1\}$ sublattice of $K$, and $\left\langle 0^{\delta}, 0^{C}\right\rangle \prec m^{\delta} \prec\left\langle 1^{\delta}, 0^{C}\right\rangle$ in $K$. 
We name a few elements of $K$ :

$$
o=\left\langle 0^{C}, 0^{C}\right\rangle ; \quad i=\left\langle 1^{C}, 1^{C}\right\rangle ; \quad a_{1}=\left\langle 0^{0}, 0^{C}\right\rangle ; \quad a_{2}=\left\langle 0^{C}, 0^{0}\right\rangle .
$$

$O$ is the zero and $i$ is the unit element of $K$. $K$ has three atoms: $a_{1}, a_{2}$, and $m\left(\left[0^{0}, 0^{C}\right],\left[0^{C}, 0^{0}\right]\right)$. Every element $x \neq 0$ of $K$ contains an atom. In fact, $K$ is strongly atomic since every chain in $K$ is well-ordered.

Without danger of confusion, we can identify the element $\left\langle x, 0^{C}\right\rangle$ of $K$ with the element $x$ of $C$. Thus, $C$ becomes a complete sublattice of $K$. Note that in (5-4), with the new notation, we get:

$$
m^{\delta} \prec 1^{\delta}, \quad \text { for } \delta<\chi ; \quad 0^{\delta} \prec m^{\delta}, \quad \text { for } \delta<\chi \text {; }
$$

and the new named elements become:

$$
o=0^{C} ; \quad a_{1}=0^{0} .
$$

It is important to observe that the interval $\left[0^{\delta},\left\langle 1^{\delta}, 0^{0}\right\rangle\right]$ of $K$ is isomorphic to $\mathfrak{M}_{X^{\delta}}$.

To sum up:

Lemma 4. $K$ is a complete lattice. It contains $C(\varphi)$ as a complete $\{0,1\}$ sublattice and $C$ as a complete sublattice. $K$ is strongly atomic. Every chain in $K$ is well-ordered. For $\delta<\chi$, attached to the appropriate part of $C$ (namely, to $\left.\left[0^{\delta}, 1^{\delta}\right]\right), K$ contains a copy of $\mathfrak{M}_{X^{\delta}}$.

\section{THE COMPLETE CONGRUENCES OF $K$}

Let $x \in L$. We are going to define a binary relation $\Theta^{x}$ on $K$. We show that the correspondence

$$
\psi: x \rightarrow \Theta^{x}
$$

is an isomorphism between $L$ and $\operatorname{Con}^{c} K$ (the lattice of complete congruences of $K$ ), proving Theorem 1 .

As a first step, we define a binary relation $\Phi^{x}$ on $C$ as follows: for $v$, $w \in C, v \leq w$,

$$
v \equiv w\left(\Phi^{x}\right) \quad \text { iff } \quad \mathfrak{p} \varphi \leq x \text { for every } \mathfrak{p} \in \operatorname{Ip}[v, w] .
$$

It is routine to check that $\Phi^{x}$ is a complete congruence on $C$. Note that $\Phi^{0}=\omega_{C}$, the zero congruence, and $\Phi^{1}=l_{C}$, the unit congruence.

Next, on $C(\varphi)$, we define (see $\S 3$ )

$$
\Theta^{x}=\Phi^{x}(\varphi)
$$

Finally, to extend $\Theta^{x}$ to $K$, we only have to make provisions for $m^{\delta}$, for $\delta<\chi$. For $y, z \in\left\{m^{\delta}, 0^{\delta}, 1^{\delta}\right\}, y \neq z$, let

$$
y \equiv z\left(\Theta^{x}\right) \text { iff } \quad 0^{\delta} \equiv 1^{\delta}\left(\Phi^{x}\right) \text { in } C .
$$


For $w \in C(\varphi)$, let

$$
\begin{aligned}
m^{\delta} \equiv w\left(\Theta^{x}\right) \quad \text { iff } \quad & 0^{\delta} \equiv 1^{\delta}\left(\Phi^{x}\right) \\
& \text { and } 1^{\delta} \equiv w\left(\Theta^{x}\right) \text { in } C(\varphi) .
\end{aligned}
$$

Lemma 5. For all $x \in L, \Theta^{x}$ is a complete congruence relation on $K$.

The verification of this lemma will be left to the reader. We give just one caution: In $C$, by $(5-2),\left[0^{C}, 0^{0}\right] \varphi=1$. Hence, the prime interval $\left[0^{\delta},\left\langle 0^{\delta}, 0^{0}\right\rangle\right]$ of $K$ is not collapsed under any $\Theta^{x}$ with $x<1$. Were this not true, Lemma 5 would fail.

Note that $\Theta^{0}=\omega_{K}$ and $\Theta^{1}=l_{K}$.

Lemma 6. $\psi$ is one-to-one and isotone.

Proof. Let $v, w \in L$. If $v \leq w$, then by (6-2), (6-3), (6-4), and (6-5), we conclude that $\theta^{v} \leq \Theta^{w}$. Hence, $\psi$ is isotone.

Let $v, w \in L-\{0\}$, and let $\Theta^{v}=\Theta^{w}$. There is a prime interval $[a, b]$ in $C$ with $[a, b] \varphi=v$, and so in $K$,

$$
a \equiv b \quad\left(\Theta^{v}\right) .
$$

Since $\Theta^{v}=\Theta^{w}$, it follows that

$$
a \equiv b \quad\left(\Theta^{w}\right) .
$$

By the definition of $\Theta^{w}=\Phi^{w}(\varphi)$ (in $\S 3$ ), it follows that, in $C$,

$$
v=[a, b] \varphi \leq w .
$$

By symmetry, $w \leq v$, implying that $v=w$. Therefore, $\psi$ is one-to-one on $L-\{0\}$. Since $\Theta^{0}=\omega$ and $\Theta^{x} \neq \omega$, for all $x \in L-\{0\}$, it follows that $\psi$ is one-to-one on $L$.

The next lemma is our first step in proving that all complete congruences $\neq \omega$ of $K$ are of the form $\Theta^{x}$, for some $x \in L-\{0\}$.

Lemma 7. Let $\mathfrak{p}$ be a prime interval of $K$. Then there exists a prime interval $\overline{\mathfrak{p}}$ of $C \subseteq K$ such that

$$
\Theta_{K}^{c}(\mathfrak{p})=\Theta_{K}^{c}(\overline{\mathfrak{p}})
$$

Proof. Let $\mathfrak{q}_{1}$ and $\mathfrak{q}_{2}$ be prime intervals in $C$. The listing $\mathfrak{p} \rightarrow \overline{\mathfrak{p}}$ given below is complete (that is, any prime interval $\mathfrak{p}$ of $K$ occurs on the left side):

$$
\begin{aligned}
\mathfrak{q}_{1} \times\{x\} \rightarrow \mathfrak{q}_{1}, & \text { for } x \in C ; \\
\{x\} \times \mathfrak{q}_{2} \rightarrow \mathfrak{q}_{2}, & \text { for } x \in C ; \\
{\left[o\left(\mathfrak{q}_{1}, \mathfrak{q}_{2}\right), m\left(\mathfrak{q}_{1}, \mathfrak{q}_{2}\right)\right] \rightarrow \mathfrak{q}_{1}, } & \text { if } \mathfrak{q}_{1} \varphi=\mathfrak{q}_{2} \varphi ; \\
{\left[m\left(\mathfrak{q}_{1}, \mathfrak{q}_{2}\right), i\left(\mathfrak{q}_{1}, \mathfrak{q}_{2}\right)\right] \rightarrow \mathfrak{q}_{1}, } & \text { if } \mathfrak{q}_{1} \varphi=\mathfrak{q}_{2} \varphi ; \\
{\left[m^{\delta}, 1^{\delta}\right] \rightarrow\left[0^{\delta}, j^{\delta}\right], } & \text { for } \delta<\chi ; \\
{\left[0^{\delta}, m^{\delta}\right] \rightarrow\left[0^{\delta}, j^{\delta}\right], } & \text { for } \delta<\chi .
\end{aligned}
$$


The first five pairs of intervals in (6-6) are in fact projective, hence,

$$
\Theta_{K}(\mathfrak{p})=\Theta_{K}(\overline{\mathfrak{p}})
$$

in particular,

$$
\Theta_{K}^{c}(\mathfrak{p})=\Theta_{K}^{c}(\overline{\mathfrak{p}})
$$

To verify the last assertion in (6-6), compute:

$$
0^{\delta} \equiv m^{\delta} \quad\left(\Theta\left(0^{\delta}, m^{\delta}\right)\right),
$$

and so

$$
\left\langle 0^{\delta}, 0^{0}\right\rangle=0^{\delta} \vee\left\langle 0^{\delta}, 0^{0}\right\rangle \equiv m^{\delta} \vee\left\langle 0^{\delta}, 0^{0}\right\rangle=\left\langle 1^{\delta}, 0^{0}\right\rangle \quad\left(\Theta\left(0^{\delta}, m^{\delta}\right)\right),
$$

yielding

$$
0^{\delta}=\left\langle 0^{\delta}, 0^{0}\right\rangle \wedge j^{\delta} \equiv\left\langle 1^{\delta}, 0^{0}\right\rangle \wedge j^{\delta}=j^{\delta} \quad\left(\Theta\left(0^{\delta}, m^{\delta}\right)\right) .
$$

This proves that

$$
\Theta\left(0^{\delta}, j^{\delta}\right) \leq \Theta\left(0^{\delta}, m^{\delta}\right)
$$

in particular,

$$
\Theta^{c}\left(0^{\delta}, j^{\delta}\right) \leq \Theta^{c}\left(0^{\delta}, m^{\delta}\right) .
$$

To prove the reverse inclusion, we start with

$$
0^{\delta} \equiv j^{\delta} \quad\left(\Theta^{c}\left(0^{\delta}, j^{\delta}\right)\right)
$$

It follows that

$$
m^{\delta}=0^{\delta} \vee m^{\delta} \equiv j^{\delta} \vee m^{\delta}=1^{\delta} \quad\left(\Theta^{c}\left(0^{\delta}, j^{\delta}\right)\right),
$$

and thus, for any $u \in\left[j^{\delta}, 1^{\delta}\right]$, we have

$$
0^{\delta}=m^{\delta} \wedge u \equiv 1^{\delta} \wedge u=u \quad\left(\Theta^{c}\left(0^{\delta}, j^{\delta}\right)\right) .
$$

By Lemma $3,1^{\delta}$ is limit, hence the complete join of these elements $u$ is $1^{\delta}$. Thus,

$$
0^{\delta} \equiv 1^{\delta} \quad\left(\Theta^{c}\left(0^{\delta}, j^{\delta}\right)\right),
$$

and so

$$
0^{\delta}=0^{\delta} \wedge m^{\delta} \equiv 1^{\delta} \wedge m^{\delta}=m^{\delta} \quad\left(\Theta^{c}\left(0^{\delta}, j^{\delta}\right)\right),
$$

completing the proof of the last case.

By Lemma 7, to investigate the congruences $\Theta^{c}(\mathfrak{p})$ of $K$, it is sufficient to take prime intervals $\mathfrak{p}$ of $C$.

Lemma 8. Let $\mathfrak{p}_{1}$ and $\mathfrak{p}_{2}$ be prime intervals of $C$ satisfying $\mathfrak{p}_{1} \varphi=\mathfrak{p}_{2} \varphi$. Then $\Theta_{K}^{c}\left(\mathfrak{p}_{1}\right)=\Theta_{K}^{c}\left(\mathfrak{p}_{2}\right)$ in $K$.

Proof. If $\mathfrak{p}_{1} \varphi=\mathfrak{p}_{2} \varphi$, then $\mathfrak{p}_{1}$ and $\mathfrak{p}_{2}$ are projective in $K$ (see Figures 1,2 , and 3 ) and the statement follows.

The following formula is the crucial step in the proof of Theorem 1: 
Lemma 9. Let $X \subseteq L-\{0\}$, and $X \neq \varnothing$. For each $x \in X \cup\{\bigvee X\}$, choose a prime interval $\mathfrak{p}_{x}$ of color $x$ of $C \subseteq K$. Then, in $K$,

$$
\Theta_{K}^{c}(\mathfrak{p} \bigvee X)=\bigvee^{c}\left(\Theta_{K}^{c}\left(\mathfrak{p}_{x}\right) \mid x \in X\right)
$$

Proof. Let $X=X^{\delta} \quad(\delta<\gamma)$. By Lemma 8, the complete congruences are not effected by which prime interval of a given color we choose. So we choose

$$
\mathfrak{p}_{\bigvee X}=\left[0^{\delta}, j^{\delta}\right] ; \quad \mathfrak{p}_{x}=\left[\left\langle 0, x_{\gamma}^{\delta}\right\rangle, u\right], \quad \text { for } x=x_{\gamma}^{\delta},
$$

where

$$
u= \begin{cases}\left\langle 0, x_{\gamma+1}^{\delta}\right\rangle, & \text { if } \gamma+1<\zeta^{\delta} \\ \left\langle 1, x_{0}^{\delta}\right\rangle, & \text { if } \gamma+1=\zeta^{\delta}\end{cases}
$$

The statement of Lemma 9 can, now, be rephrased as follows:

In $K$,

$$
\Theta_{K}^{c}\left(0^{\delta}, j^{\delta}\right)=\bigvee^{c}\left(\Theta_{K}^{c}\left(\left\langle 0, x_{\gamma}^{\delta}\right\rangle, u_{\gamma}^{\delta}\right) \mid \gamma<\zeta^{\delta}\right),
$$

where $u_{\gamma}^{\delta}$ is defined in (6-9).

As we pointed out in $\S 5$ (see Lemma 4), the interval $\left[0^{\delta},\left\langle 1^{\delta}, 0^{0}\right\rangle\right]$ is isomorphic to $\mathfrak{M}_{X^{\delta}}$. Hence the following statement is a stronger form of $(6-10)$ :

In $\mathfrak{M}_{X^{\delta}}$

$$
\Theta_{\mathfrak{M}_{X^{\delta}}}^{c}\left(0^{\delta}, j^{\delta}\right)=\bigvee^{c}\left(\Theta_{\mathfrak{M}_{X^{\delta}}}^{c}\left(\left\langle 0, x_{\gamma}^{\delta}\right\rangle, u_{\gamma}^{\delta}\right) \mid \gamma<\zeta^{\delta}\right),
$$

where $u_{\gamma}^{\delta}$ is defined in (6-9).

To prove (6-11), consider in $\mathfrak{M}_{X^{\delta}}$ the well-ordered chain $Q$ :

$$
j^{\delta}=\left\langle 0, x_{0}^{\delta}\right\rangle<\left\langle 0, x_{1}^{\delta}\right\rangle<\cdots<\left\langle i, x_{0}^{\delta}\right\rangle<\left\langle i, x_{1}^{\delta}\right\rangle<\cdots<\left\langle i, x_{\gamma}^{\delta}\right\rangle<\cdots,
$$

where $i<\omega$ and $\gamma<\zeta^{\delta}$. Each prime interval of this chain,

$$
\begin{array}{ll}
{\left[\left\langle i, x_{\gamma}^{\delta}\right\rangle,\left\langle i, x_{\gamma+1}^{\delta}\right\rangle\right],} & \text { for } i<\omega, \gamma<\zeta^{\delta}, \\
{\left[\left\langle i, x_{\gamma}^{\delta}\right\rangle,\left\langle i+1, x_{0}^{\delta}\right\rangle\right],} & \text { for } i<\omega, \gamma+1=\zeta^{\delta},
\end{array}
$$

is of color $x$, for some $x \in X$-see (4-3) and (4-4). Thus

$$
\Theta=\bigvee^{c}\left(\Theta_{K}^{c}\left(\mathfrak{p}_{x}\right) \mid x \in X\right)
$$

collapses all the prime intervals of $Q$. Since $Q$ is a well-ordered chain, $1^{\delta}$ is a limit, $Q \cup\left\{1^{\delta}\right\}$ is a complete sublattice of $\mathfrak{M}_{X^{\delta}}$, and $\Theta$ is a complete congruence, it follows that $\Theta$ collapses $\left[j^{\delta}, 1^{\delta}\right]$. Thus,

$$
\Theta=\bigvee^{c}\left(\Theta_{\mathfrak{M}_{X^{\delta}}}^{c}\left(\mathfrak{p}_{x}\right) \mid x \in X\right)=\Theta_{\mathfrak{M}_{X^{\delta}}}^{c}\left(j^{\delta}, 1^{\delta}\right)
$$

Now,

$$
j^{\delta} \equiv 1^{\delta} \quad\left(\Theta_{\mathfrak{M}_{\chi^{\delta}}}^{c}\left(j^{\delta}, 1^{\delta}\right)\right) ;
$$


meeting with $\mathrm{m}^{\delta}$, we get that

$$
0^{\delta} \equiv m^{\delta} \quad\left(\boldsymbol{\Theta}_{\mathfrak{M}_{X^{\delta}}}^{c}\left(j^{\delta}, 1^{\delta}\right)\right)
$$

Joining with $\left\langle 0^{\delta}, 1\right\rangle$, yields that

$$
\left\langle 0^{\delta}, 1\right\rangle \equiv\left\langle 1^{\delta}, 1\right\rangle \quad\left(\Theta_{\mathfrak{M}_{X^{\delta}}}^{c}\left(j^{\delta}, 1^{\delta}\right)\right) .
$$

Finally, meeting with $j^{\delta}$, we obtain that

$$
0^{\delta} \equiv j^{\delta} \quad\left(\boldsymbol{\Theta}_{\mathfrak{M}_{X^{\delta}}}^{c}\left(j^{X}, 1^{\delta}\right)\right) .
$$

Therefore,

$$
\bigvee^{c}\left(\Theta_{\mathfrak{M}_{X^{\delta}}}^{c}\left(\mathfrak{p}_{x}\right) \mid x \in X\right)=\Theta_{\mathfrak{M}_{X^{\delta}}}^{c}\left(j^{\delta}, 1^{\delta}\right) \geq \Theta_{\mathfrak{M}_{X^{\delta}}}^{c}\left(0^{\delta}, j^{\delta}\right)=\Theta_{\mathfrak{M}_{X^{\delta}}}^{c}\left(\mathfrak{p}_{\bigvee}\right)
$$

proving $\leq$ in (6-11). Since $\geq$ in (6-11) follows from Lemma 6, this completes the proof of the lemma.

Applying Lemma 9 to the special case $X=\{a, b\}, 0<a \leq b$ in $L$, and to two prime intervals $\mathfrak{p}_{1}, \mathfrak{p}_{2}$ of $C \subseteq K$, with $\mathfrak{p}_{1} \varphi=a$ and $\mathfrak{p}_{2} \varphi=b$, we conclude that

Lemma 10. $\mathfrak{p}_{1} \varphi \leq \mathfrak{p}_{2} \varphi$ in $L$ implies that $\Theta_{K}^{c}\left(\mathfrak{p}_{1}\right) \leq \Theta_{K}^{c}\left(\mathfrak{p}_{2}\right)$ in $K$.

Now we are ready to tackle complete congruences generated by prime intervals:

Lemma 11. Let $\mathfrak{p}$ be a prime interval in $C \subseteq K$. Then

$$
\Theta_{K}^{c}(\mathfrak{p})=\Theta^{\mathfrak{p} \varphi} \text {. }
$$

Proof. Lemma 10 states that $\Theta_{K}^{c}(\mathfrak{p})$ collapses all prime intervals $\mathfrak{p}_{1}$ of $C$ satisfying $\mathfrak{p}_{1} \varphi \leq \mathfrak{p} \varphi$. Thus $\theta_{K}^{c}(\mathfrak{p})$ defines the relation $\Phi^{\mathfrak{p} \varphi}$ of $(6-2)$ on $C$ which was used to define $\Theta^{p \varphi}$ in (6-3), (6-4), and (6-5), hence the equality.

Now we are ready for the final step:

Lemma 12. All complete congruences $\neq \omega$ of $K$ are of the form $\Theta^{x}$, for some $x \in L-\{0\}$.

Proof. Let $\Phi$ be a complete congruence of $K$. Since $K$ is strongly atomic (Lemma 4), by Lemma 1 , pi $\Phi=\Phi$, and so

$$
\Phi=\bigvee^{c}\left(\Theta^{c}(\mathfrak{p}) \mid \mathfrak{p} \in \operatorname{Ip} K, \quad \Theta(\mathfrak{p}) \leq \Phi\right)
$$

Thus, by Lemma 7,

$$
\Phi=\bigvee^{c}\left(\Theta^{c}(\mathfrak{p}) \mid \mathfrak{p} \in \operatorname{Ip} C, \boldsymbol{\Theta}(\mathfrak{p}) \leq \Phi\right) .
$$

By Lemma 11, it follows from this formula that

$$
\Phi=\bigvee^{c}\left(\Theta^{x} \mid x \in X\right)
$$


where $X=\{\mathfrak{p} \varphi \mid \mathfrak{p} \in \operatorname{Ip} C, \Theta(\mathfrak{p}) \leq \Phi\}$. By Lemma $7, X \neq \varnothing$. Now we apply (6-7), and we obtain

$$
\Phi=\Theta^{\bigvee x}
$$

completing the proof.

By Lemma 6, $\psi$ is one-to-one; by Lemma $9, \psi$ preserves joins (in fact, infinite joins). By Lemma 12 and the observation that $\omega_{K}=\Theta^{0}$, any complete congruence $\Phi$ of $K$ can be represented as $\Phi=\Theta^{x}$ for some $x \in L$; thus, $\psi$ is onto, proving that $\psi$ is an isomorphism between $L$ and $\operatorname{Com}^{c} K$, completing the proof of Theorem 1 .

\section{Proof of Theorem 2}

Based on the results of R. Frucht [4] and G. Sabidussi [21] (see, also, A. Pultr and V. Trnková [19]), it is routine to see that we can represent the group $G$ as the automorphism group of a connected undirected graph $\mathfrak{G}=\langle V, E\rangle$ without loops, where $V$ is the set of vertices and $E$ is the set of edges.

Next, we represent $G$ by a bounded lattice and lattice automorphisms. As in Frucht [5], from $\mathfrak{G}$ we form the lattice:

$$
H=V \dot{\cup} E \dot{\cup}\{0,1\},
$$

where $0<v<1,0<e<1$, for all $v \in V$ and $e \in E$; let $v<e$ in $H$ iff $v \in e$. Note that $H$ is of length three, and therefore complete.

The graphs constructed in Frucht [5] have the following property:

(7-1) For $v \in V$, there are $e_{1}, e_{2} \in E$ with $v \not e_{1}, e_{2}$ and $e_{1} \cap e_{2}=\varnothing$.

It is easy to prove that if the graph $\mathfrak{G}$ has property (7-1), then the associated lattice is simple. Hence, $H$ is a simple lattice.

We attach $H$ to $K$ by identifying the unit element $i$ of $K$ with the zero $0_{H}$ of $H$; we add a complement $q$ of $i$ (see Figure 10). The next three lemmas show that the resulting lattice $K_{H}$ will do the job.

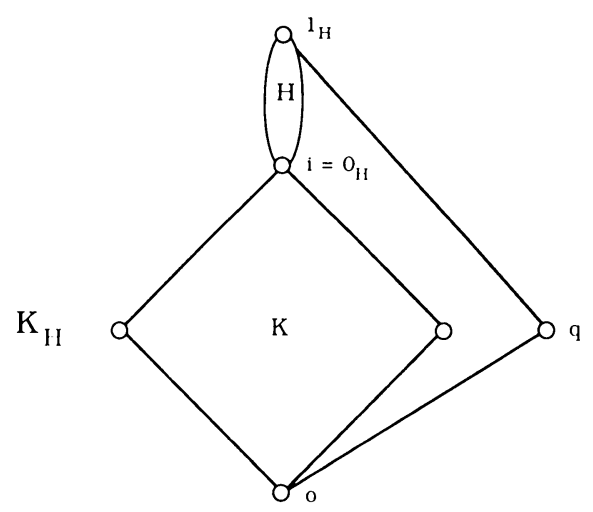

FIGURE 10 
Let $\Theta$ be a complete congruence relation of $K$. We shall define an extension $\bar{\Theta}$ of $\Theta$ to $K_{H}$ : If $\Theta<l_{K}$, let $\bar{\Theta}$ be the congruence relation of $K_{H}$ that is $\Theta$ on $K$ and trivial outside of $K$. More formally,

$(7-2) x \equiv y(\bar{\Theta})$ iff $x=y$ or $x, y \in K$ and $x \equiv y$

for $x, y \in K_{H}$. We define $\bar{\imath}_{K}=l_{K_{H}}$.

Lemma 13. The complete congruence relations of $K_{H}$ are the relations of the form $\bar{\Theta}$, where $\Theta$ is a complete congruence of $K$.

Proof. The proof is by straightforward computation. Note only that if $\Theta<l$, then, in $K,\{o\}$ is a congruence class of $\Theta$; this is why the extension $\bar{\Theta}$ can be defined to be trivial outside of $K$. Since $H$ is simple, it follows that all complete congruences of $K_{H}$ extend from $K$.

Lemma 13 immediately implies that the complete congruence lattice of $K_{H}$ is isomorphic to the complete congruence lattice of $K$.

Now, for the automorphisms.

Lemma 14. $K$ has no nontrivial automorphism.

Proof. Let $\alpha$ be an automorphism of $K$. Under $\alpha$, the image of a meetreducible atom is a meet-reducible atom; therefore, $a_{1} \alpha=a_{1}$ or $a_{1} \alpha=a_{2}$. The latter is impossible since, in $K,\left[a_{1}\right)$ is nonmodular while $\left[a_{2}\right)$ is modular. Hence,

$$
a_{1} \alpha=a_{1} \text { and } a_{2} \alpha=a_{2} .
$$

The elements $1^{C}\left(=\left\langle 1^{C}, 0^{C}\right\rangle\right)$ and $\left\langle 0^{C}, 1^{C}\right\rangle$ are the only doubly-irreducible and completely join-reducible elements in $K$; since $a_{1} \alpha=a_{1}$, it follows that

$$
1^{C} \alpha=1^{C} \text { and }\left\langle 0^{C}, 1^{C}\right\rangle \alpha=\left\langle 0^{C}, 1^{C}\right\rangle \text {. }
$$

Therefore, the interval $\left[a_{1}, 1^{C}\right]$ is mapped into itself by $\alpha$ and so the meetreducible elements of the interval, that is, the elements of the form $\left\langle 0^{C}, x\right\rangle$, $x \in C$, are mapped into themselves. We conclude that $\alpha$ can be regarded as an automorphism of $C$. Since $C$ is a well-ordered set, it has no nontrivial automorphism and so $\alpha$ is the identity map on $C$. Arguing similarly, we get that $\alpha$ is the identity map on $\left\{\left\langle 0^{C}, x\right\rangle \mid x \in C\right\}$. Therefore, $\alpha$ is the identity map on $C \times C$. It now easily follows that $\alpha$ is the identity map on $C(\varphi)$ and on $K$. This completes the proof of the lemma.

Now, let $\alpha$ be an automorphism of $H$. We extend $\alpha$ to $K_{H}$ trivially:

$$
x \bar{\alpha}= \begin{cases}x \alpha, & \text { if } x \in H \\ x, & \text { otherwise. }\end{cases}
$$

Lemma 15. Let $\alpha$ be an automorphism of $H$. Then $\bar{\alpha}$ is an automorphism of $K_{H}$. Conversely, every automorphism of $K_{H}$ can be uniquely represented in this form.

Proof. Let $\beta$ be an automorphism of $K_{H}$. Observe that $0_{H}=i$ is the only element $u$ of $K_{H}$ with the property that there is a maximal chain of length three 
in $\left[u, 1^{H}\right]$. Hence, $i \beta=i$. Thus $\beta$ induces an automorphism $\beta_{K}$ of $K$ and an automorphism $\beta_{H}$ of $H$. By Lemma $14, \beta_{K}$ is the identity map. Define $\alpha=\beta_{H}$. Then $\beta=\bar{\alpha}$, as defined in (7-3), as claimed. This representation is obviously unique, completing the proof of the lemma.

Lemma 15 obviously implies that the automorphism group of $K_{H}$ is isomorphic to $G$. Therefore, Lemmas 13 and 15 prove Theorem 2 .

\section{CONCLUding COMMENTS}

We see from this paper how differently complete congruences of a complete lattice and congruences of a lattice behave. If $K$ is a lattice and $\mathfrak{p}$ is a prime interval of $K$, then $\Theta(p)$ is a join-irreducible element of Con $K$. The complete lattice $K$ constructed in the proof of Theorem 1 has the property that every $\Theta>\omega$ is generated by a prime interval; that is, for every complete congruence $\Theta$ other than the trivial congruence $\omega$ there is a prime interval $\mathfrak{p}$ of $K$ such that $\Theta=\Theta^{c}(\mathfrak{p})$.

We have already utilized in Lemma 13 another property of $K$ : If $\Theta$ is a complete congruence of $K$ and $\Theta<l$, then $\{o\}$ is a complete congruence class of $\Theta$. To prove the result announced in [9], we also need the dual property. Let us state the properties of $K$ formally:

Theorem $\mathbf{1}^{\prime}$. For every complete lattice $L$, there is complete lattice $K$ with zero and unit elements $O$ and $i$, respectively, with the following properties:

(1) $L$ is isomorphic to the lattice of complete congruence relations of $K$.

(2) For every complete congruence $\Theta$ of $K$ other than the trivial congruence $\omega$, there is a prime interval $\mathfrak{p}$ of $K$ such that $\Theta=\Theta^{c}(\mathfrak{p})$.

(3) For any complete congruence $\Theta$ of $K$, if $\Theta<l$, then $\{o\}$ is a complete congruence class of $\Theta$.

(4) For any complete congruence $\Theta$ of $K$, if $\Theta<l$, then $\{i\}$ is a complete congruence class of $\boldsymbol{\Theta}$.

(5) $K$ has no nontrivial automorphism.

Proof. To satisfy (4), we have to add a dual atom $m^{C}$ to $C$, and color the new prime interval by $1:\left[\mathrm{m}^{C}, 1^{C}\right] \varphi=1$. The only problem is that the resulting lattice $K^{\prime}$ fails to satisfy (5). It is easy to see that by removing from $K^{\prime}$ two elements, $\left\langle 1^{C}, 0^{C}\right\rangle$ and $\left\langle 0^{C}, 1^{C}\right\rangle$, we obtain a lattice $K^{\prime \prime}$ satisfying all five properties.

Alternatively, in the construction of $K$, we can choose $\chi$ as a successor ordinal, $\xi+1$, and choose $X^{\xi} \subseteq L-\{0\}$ so that $\bigvee X^{\xi}=1$. Then $K$ satisfies property (4).

From the point of view of infinitary universal algebras, Theorem 1 is very pleasing; it represents every complete lattice $L$ as the lattice of complete congruence relations of a suitable complete lattice $K$, while Grätzer and Lampe [13] represented $L$ as the lattice of congruence relations of an infinitary universal algebra of unspecified type. Since one can view a complete lattice $L$ as 
an infinitary algebra, the new result contains the old. However, it should be pointed out that we do not get a uniform type. In fact, it was proved in 1979 by R. Freese, Lampe, and W. Taylor [3] that it is not possible to have a uniform type of finitary universal algebras in the finitary congruence lattice characterization theorem. The analogous result for infinitary algebras is trivial to prove. In contrast, what we get in Theorem 1 is a family of types associated with complete lattices.

It seems like an interesting question whether there is a natural family of finitary types that would admit a uniform representation of congruence lattices of finitary universal algebras.

In Grätzer and Lakser [11], we present a general result, the One Point Extension Theorem, concerning extending lattices by an element for every interval in a given family of intervals. The One Point Extension Theorem gives conditions under which congruences extend, and extend uniquely. A number of special cases of this result are contained in the present paper: the statement in the paragraph following (3-2), Lemmas 2, 5, and 13.

Some of the results of this paper generalize to $\mathfrak{M}$-complete lattices; these results were announced in [10] and written up in [11].

Theorem $1^{\prime}$ has an interesting application to infinitary universal algebra announced in [9]: a new proof of the independence of the congruence lattice, the subalgebra lattice, and the automorphism group of an infinitary universal algebra. This independence result was first announced in [13] (see Appendix 7 of [7] for a complete proof). While the proof of Grätzer and Lampe apply to both the finitary and infinitary case, the new simpler proof only does the infinitary case.

\section{REFERENCES}

1. G. Birkhoff, Universal algebra, Proc. First Canadian Math. Congress, Montreal, 1945, Univ. of Toronto Press, Toronto, 1946, pp. 310-326.

2. __ Lattice theory, Amer. Math. Soc. Colloq. Publ., vol. 25, revised ed., Amer. Math. Soc., Providence, R.I., 1948.

3. R. Freese, W. A. Lampe, and W. Taylor, Congruence lattices of algebras of fixed similarity types. I, Pacific J. Math. 82 (1979), 59-68.

4. R. Frucht, Herstellung von Graphen mit vorgegebener abstrakter Gruppe, Compositio Math. 6 (1938), 239-250.

5. __ Lattices with a given group of automorphisms, Canad. J. Math. 2 (1950), 417-419.

6. G. Grätzer, General lattice theory, Academic Press, New York, 1978; Birkhäuser Verlag, Basel; Akademie Verlag, Berlin.

7. __ Universal algebra, 2nd ed., Springer-Verlag, New York, Heidelberg, Berlin, 1979.

8. lattice, Abstracts Amer. Math. Soc. 9 (1988), 416. Abstract 88T-06-215.

9. __ A "lattice theoretic" proof of the independence of the automorphism group, the congruence lattice, and subalgebra lattice of an infinitary algebra, Abstracts Amer. Math. Soc. 9 (1988), 503. Abstract 88T-08-254. 
10. G. Grätzer and $\mathrm{H}$. Lakser, On the $\mathfrak{M}$-complete congruence lattice and the automorphism group of an $\mathfrak{M}$-complete lattice, Abstracts Amer. Math. Soc. 9 (1988), 503. Abstract 88T-08253.

11. _ On congruence lattices of $\mathfrak{M}$-complete lattices, Preprint, University of Manitoba.

12. G. Grätzer, H. Lakser, and B. Wolk, On the lattice of complete congruences of a complete lattice: On a result of $K$. Reuter and $R$. Wille, Acta Sci. Math. (Szeged) (to appear).

13. G. Grätzer and W. A. Lampe, Representations of complete lattices as congruence lattices of infinitary algebras. I, II, III, Notices Amer. Math. Soc. 18, 19 (1971-1972), 937, A-683, A-749.

14. G. Grätzer and E. T. Schmidt, On congruence lattices of lattices, Acta Math. Acad. Sci. Hungar. 13 (1962), 179-185.

15. Characterizations of congruence lattices of abstract algebras, Acta Sci. Math. (Szeged) 24 (1963), 34-59.

16. W. A. Lampe, On the congruence lattice characterization theorem, Trans. Amer. Math. Soc. 182 (1973), 43-60.

17. E. Nelson, The independence of the subalgebra lattice, congruence lattice, and automorphism group of an infinitary algebra, J. Pure Appl. Algebra 17 (1980), 187-201.

18. P. Pudlák, A new proof of the congruence lattice representation theorem, Algebra Universalis 6 (1976), 269-275.

19. A. Pultr and V. Trnková, Combinatorial, algebraic and topological representations of groups, semigroups and categories, Academia, Prague, 1980.

20. K. Reuter and R. Wille, Complete congruence relations of complete lattices, Acta Sci. Math. (Szeged) 51 (1987), 319-327.

21. G. Sabidussi, Graphs with given infinite groups, Monatsch. Math. 64 (1960), 64-67.

22. E. T. Schmidt, Kongruenzrelationen algebraischer Strukturen, Math. Forschungberichte, XXV, VEB Deutscher Verlag der Wissenschaften, Berlin, 1967.

23. S.-K. Teo, Representing finite lattices as complete congruence lattices of complete lattices, Abstracts Amer. Math. Soc. 9 (1988), 416. Abstract 88T-06-207; Ann. Univ. Sci. Budapest. Eötvös Sect. Math. (to appear).

24. R. Wille, Subdirect decompositions of concept lattices, Algebra Universalis 17 (1983), 275287.

Department of Mathematics, University of Manitoba, Winnipeg, Manitoba R3T 2N2, CANADA 